\title{
Self-reported lactose intolerance in clinic patients with functional gastrointestinal symptoms: prevalence, risk factors, and impact on food choices
}

Zheng, X ; Chu, H ; Cong, Y ; Deng, Y ; Long, Y ; Zhu, Y ; Pohl, D ; Fried, M ; Dai, N ; Fox, M

\begin{abstract}
BACKGROUND: Many patients complain of abdominal symptoms with dairy products; however, clinical and psychosocial factors associated with self-reported lactose intolerance (SLI) have not been assessed in large studies. In particular, data are lacking from lactase deficient populations. This prospective cohort study assessed the prevalence of, and risk factors for, SLI in Chinese patients attending a gastroenterology clinic. METHODS: Consecutive patients completed questionnaires to assess digestive health (Rome III), psychological state (HADS), life event stress (LES), food intake, and quality-of-life (SF-8). A representative sample completed genetic studies and hydrogen breath testing (HBT) at the clinically relevant dose of $20 \mathrm{~g}$ lactose. KEY RESULTS: SLI was present in 411/910 (45\%) clinic patients with functional abdominal symptoms. The genotype in all subjects was C/C-13910. A small number of novel SNPs in lactase promoter region were identified, including C/T-13908 which appeared to confer lactase persistence. Over half of the patients (54\%) completed the $20 \mathrm{~g}$ lactose HBT with 58\% (285/492) reporting typical symptoms. Positive and negative predictive values of SLI for abdominal symptoms during HBT were $60 \%$ and 44\%, respectively. Psychological state and stress were not associated with SLI in clinic patients. SLI impacted on physical quality-of-life and was associated with reduced ingestion of dairy products, legumes, and dried fruit ( $\mathrm{p}$ 0.05). CONCLUSIONS INFERENCES: In a lactase deficient population, approximately half of patients attending clinic with functional gastrointestinal symptoms reported intolerance to dairy products; however, SLI did not predict findings on $20 \mathrm{~g}$ lactose HBT. Independent of psychosocial factors, SLI impacted on quality-of-life and impacted on food choices with restrictions not limited to dairy products.
\end{abstract}

DOI: https://doi.org/10.1111/nmo.12602

Posted at the Zurich Open Repository and Archive, University of Zurich ZORA URL: https://doi.org/10.5167/uzh-119675

Journal Article

Accepted Version

Originally published at:

Zheng, X; Chu, H; Cong, Y; Deng, Y; Long, Y; Zhu, Y; Pohl, D; Fried, M; Dai, N; Fox, M (2015). Self-reported lactose intolerance in clinic patients with functional gastrointestinal symptoms: prevalence, risk factors, and impact on food choices. Neurogastroenterology and Motility, 27(8):1138-1146.

DOI: https://doi.org/10.1111/nmo.12602 
Title page

Self-reported lactose intolerance in clinic patients with functional gastrointestinal symptoms: prevalence, risk factors and impact on food choices

Running head: self-reported lactose intolerance

Authors: Xia Zheng ${ }^{1,}$ Hua Chu ${ }^{1}$, Yanqun Cong ${ }^{1}$, Yanyong Deng ${ }^{1}$, Yanqin Long ${ }^{1}$, Yubin Zhu ${ }^{1}$, Daniel Pohl ${ }^{2,3}$, Michael Fried $^{2,3}$, Ning Dai $^{1^{*}}$, Mark Fox $^{2,3^{*}}$

\section{Author affiliations:}

${ }^{1}$ Department of Gastroenterology, Sir Run Run Shaw Hospital, School of Medicine, Zhejiang University, Hangzhou, China;

${ }^{2}$ Division of Gastroenterology \& Hepatology, University Hospital Zürich, Zürich, Switzerland;

${ }^{3}$ Zürich Centre for Integrative Human Physiology (ZIHP), University of Zürich, Zürich, Switzerland.

Co-corresponding authors: Ning Dai, Mark Fox

Dr. Ning Dai, Department of Gastroenterology, Sir Run Run Shaw Hospital, School of Medicine, Zhejiang University, \#3 East Qingchun Road, Hangzhou 310016,China. Tel: 86-0571-86096182, Fax: 86-0571-86044817; dainingcn@gmail.com;

Dr. Mark Fox, Division of Gastroenterology \& Hepatology, University Hospital Zürich, CH-8091 Zürich, Switzerland. d.mark.fox@gmail.com 


\section{Self-reported lactose intolerance in clinic patients with functional gastrointestinal} symptoms: prevalence, risk factors and impact on food choices

\section{ABSTRACT}

Background: Many patients complain of abdominal symptoms with dairy products; however, clinical and psychosocial factors associated with self-reported lactose intolerance (SLI) have not been assessed in large studies. In particular, data is lacking from lactase deficient populations. This prospective cohort study assessed the prevalence of, and risk factors for, SLI in Chinese patients attending a gastroenterology clinic.

Methods: Consecutive patients completed questionnaires to assess digestive health (Rome III), psychological state (HADS), life event stress (LES), food intake and quality-of-life (SF-8). A representative sample completed genetic studies and hydrogen breath testing (HBT) at the clinically relevant dose of $20 \mathrm{~g}$ lactose.

Key Results: SLI was present in 410/910 (45\%) clinic patients with functional abdominal symptoms. The genotype in all subjects was C/C-13910. A small number of novel SNPs in lactase promoter region were identified, including C/T-13908 which appeared to confer lactase persistence. Over half of the patients (54\%) completed the 20g lactose HBT with 58\% (285/492) reporting typical symptoms. Positive and negative predictive values of SLI for abdominal symptoms during HBT were $60 \%$ and $44 \%$, respectively. Psychological state and stress were not associated with SLI in clinic patients. SLI impacted on physical quality-of-life and was associated with reduced ingestion of dairy products, legumes and dried fruit $(\mathrm{p} \leq 0.05)$

Conclusions \& Inferences: In a lactase deficient population, approximately half of patients 
attending clinic with functional gastrointestinal symptoms reported intolerance to dairy products; however, SLI did not predict findings on $20 \mathrm{~g}$ lactose HBT. Independent of psychosocial factors, SLI impacted on quality-of-life and impacted on food choices with restrictions not limited to diary products.

\section{Key Messages:}

- In a lactase deficient population, approximately half of patients attending clinic with functional gastrointestinal symptoms reported intolerance to dairy products.

- $\quad$ SLI did not predict the occurrence of symptoms by $20 \mathrm{~g}$ Lactose HBT.

- $\quad$ SLI subjects avoided lactose intake from dairy products and reduced intake also of other foods, such as legumes and dried fruit, known to cause bloating and flatulence.

- Independent of psychosocial factors, the presence of food intolerance impacted on quality-of-life.

Key Words: FODMAPs; hydrogen breath test; lactase deficiency; lactose intolerance; psychological state.

Abbreviations: HBT, hydrogen breath test; HADS, hospital anxiety and depression scale;

LM, lactose malabsorption; LI, lactose intolerance; LEST, life event stress test; SLI, self-reported lactose intolerance; SNP, single nucleotide polymorphism; TSS, total symptom score. 


\section{INTRODUCTION}

Lactose is the major carbohydrate found in milk and dairy products and is used widely by the food industry in processed foods. Lactase deficiency leads to lactose malabsorption (LM) with passage of undigested nutrient into the colon. ${ }^{1,2}$ Lactose intolerance (LI) refers to digestive symptoms such as bloating, diarrhea and abdominal pain that occur as a result of the production of gas and other products of bacterial fermentation in the digestive tract.

The presence of lactase deficiency on genetic sequencing or lactose malabsorption on hydrogen breath tests (HBT) does not automatically mean that patients report clinically relevant symptoms after ingestion of lactose at the level found in the normal diet. ${ }^{3,4}$ Indeed, 9 in 10 individuals with lactase deficiency tolerate a cup of milk (10g lactose) and 1 in 5 can take up to a liter of milk ( $40 \mathrm{~g}$ lactose) without reporting symptoms. ${ }^{5-7}$ Conversely others, often those with functional gastrointestinal symptoms (e.g. dyspepsia, irritable bowel syndrome), claim that ingestion of dairy products causes symptoms when there is no evidence of lactose malabsorption. ${ }^{7,8}$ On this basis, controlled tests of food tolerance at clinically relevant doses have been recommended to ensure that any advice to restrict dairy products is based on objective rather than subjective evidence. ${ }^{9}$

Recently we have identified demographic, psycho-social and clinical risk factors for food intolerance at clinically relevant doses (20g lactose HBT) in a Chinese population with primary lactase deficiency: an in-vivo model of food intolerance in humans. ${ }^{5,10}$ Extrinsic factors related to lactose intolerance included lactose dose, small intestinal bacterial overgrowth and high levels of $\mathrm{H} 2$ production from the colonic microbiota. Patient factors such as anxiety, stress and the presence of functional gastrointestinal disease were also 
associated with food intolerance. $5,10,11$

The association between self-reported lactose intolerance (SLI) and objective evidence of lactose malabsorption intolerance on HBT at any dose was weak. ${ }^{5}$ This is important because individuals with SLI may limit intake of dairy products and may avoid other products thought to cause abdominal symptoms. ${ }^{12}$ The presence of multiple food intolerances can impact on quality of life. ${ }^{13}$ Multiple dietary exclusions can lead to a highly restrictive diet deficient in vital micronutrients. For example, self-imposed reductions in consumption of dairy products have been linked to reduced calcium intake, increased bone turnover and, ultimately, an increased risk of osteoporosis and fracture. ${ }^{14,15}$ SLI has also been associated with other conditions such as diabetes and hypertension, ${ }^{16}$ although it remains uncertain whether the latter associations are related to reduced intake of dairy products or whether this is a non-specific consequence of lifestyle and food choices.

These concerns highlight the need to identify patients with self-reported food intolerance that are making inappropriate food choices that could impact on nutritional health and quality of life. This prospective cohort study aimed to determine the prevalence and risk factors for SLI in a large group of adult patients attending a gastroenterology clinic. The association between objective and self-reported assessment of lactose malabsorption and intolerance was assessed. The impact of SLI on quality of life and food choices was documented.

\section{METHODS}

\section{Subjects and study design}

The present study was conducted in Sir Run Run Shaw Hospital in Hangzhou in the 
South-Eastern China. Consecutive patients aged 16-74 with lower digestive tract symptoms without alarm symptoms (i.e. dysphagia, anaemia, overt blood loss, abdominal mass and weight loss) attending the morning gastrointestinal clinic were included and completed a series of questionnaires via face-to-face interview to assess the prevalence of functional gastrointestinal disease as defined by the Rome III criteria, psychosocial status, nutritional intake and quality of life (see next section). As is typical in the Chinese health system, more than $90 \%$ of patients attending hospital clinics were self-referrals (i.e. hospitals provide a primary care service).

Participants with a past history of organic gastrointestinal disease (e.g. peptic ulcer, inflammatory bowel disease), diabetes mellitus, thyroid disease, drug abuse and alcohol addiction were excluded. Individuals with a history of abdominal surgery except appendectomy, cholecystectomy and hernia repair were also excluded. All participants signed a written informed consent form and the study was approved by the ethical committee of Sir Run Run Shaw Hospital and registered (ClinicalTrials.gov: NCT01286597). Studies were conducted in accordance with the Declaration of Helsinki and written informed consent was obtained from all participants.

\section{Questionnaires}

All questionnaires were completed under supervision of investigators to ensure participants understood questions and responded to all questions.

1. SF-8: Short version of the SF-36 questionnaire documenting quality of life in 8 domains: physical functioning, role-physical, bodily pain, general health, vitality, social 
functioning, role-emotional and mental health. Higher scores indicate a better health-related quality of life.

2. FBD: Rome III questionnaire to diagnose functional gastrointestinal disease.

3. HADS (Hospital anxiety and depression scale): HADS score $\geq 11$ was taken as clinically significant level of anxiety or depression, with a cut-off score $\geq 8$ for diagnosing borderline neurosis. For the purposes of categorical analysis anxiety or depression was defined as a score of $\geq 8$ for each respective scale in our study.

4. LEST (Life Event Stress Test): The social readjustment rating scale measuring stressful events in life. ${ }^{17}$ Culturally specific items (e.g. currency) were translated. A sum score was computed using the weights given in the cited publication.

5. FFQ: Food intake frequency and portion sizes was recorded using a standardized questionnaire validated in a Chinese population ${ }^{18}$. This FFQ is a list of 30 foods, including dairy products: milk, yoghurt, flavored milk, milk-powder, condensed milk. Non-dairy foods included bread, cake, biscuit, sausage, chocolate, ice-cream and cheese. Daily lactose intake from milk products was recorded separately from lactose in other food sources and the total amount of lactose calculated by reference to the USDA National Nutrient Database (http://www.nal.usda.gov/fnic/foodcomp/search/, accessed on October 4, 2012).

6. Self-reported Lactose Intolerance (SLI) was present in an individual if he or she responded "yes" to the question "Do you have abdominal discomfort such as abdominal pain, bloating, diarrhea after intake of milk or dairy products?" People who responded "no" or “don't know" were classified as not having SLI.

7. General information: Demographic data of subjects (age, gender, marital status, 
education, profession, income range), smoking and alcohol consumption, past medical and surgical history.

\section{Lactose hydrogen breath test}

Patients that attended gastroenterology clinic in the morning completed a $20 \mathrm{~g}$ lactose hydrogen breath test (HBT, equivalent to $400 \mathrm{ml}$ milk) validated for use in populations with a high rate of lactase deficiency. ${ }^{5,11}$ This was not feasible for patients attending in the afternoon due to the 3 hours duration of the test. Exhaled hydrogen was recorded every 15 min for $3 \mathrm{~h}$ by a portable analyzer. LM was diagnosed if peak $\mathrm{H} 2$ breath excretion exceeded $\geqslant 20 \mathrm{ppm}$ over the baseline value on at least 2 consecutive readings. The number and the severity of individual symptoms (nausea, bloating, abdominal pain, borborygmi and diarrhea) during and after the test within $24 \mathrm{~h}$ were assessed by a Likert scale $(0=$ absence, $1=$ trivial, $2=$ mild, 3=moderate, $4=$ strong, $5=$ severe symptoms). The total symptom score (TSS) was calculated as the sum of the highest intensity value for each symptom. ${ }^{19}$ LI was diagnosed if, in the presence of LM, a greater than 1 point rise in TSS was reported on at least two consecutive measurements. 5,10

\section{Genetic sequencing of lactase gene regulatory sequence (genotype)}

A large sample of clinic patients completed genetic testing for primary lactase deficiency (i.e. sequencing of lactase gene regulatory sequence). Reference data from healthy volunteers was on file. White cells were isolated from a whole blood sample using a modified salting-out procedure, and DNA was extracted (Axygen, Union City, CA, USA). A 446-bp region within 
intron 13 of MCM6 (13807bp to $14253 \mathrm{bp}$ upstream of the lactase gene) was amplified by 35 PCR cycles (forward primer (5'-CGGATGCACTGCTGTGATGA-3'), reverse primer (5'-ACTGACCTATCCTCGTGGAATG-3')). Single Nucleotide Polymorphisms (SNPs) associated with lactase persistence in European (C/T-13910), African and Arabian (C/G-13907, T/G-13915, G/C-14010) populations were identified by bi-directional sequencing using Sequencher software (vs. 4.0.5, GeneCodes). ${ }^{20,21}$

\section{Statistical analysis}

The appropriate number of participants to recruit was estimated based on published data from the South Chinese population that reported (i) $\sim 80 \%$ and $\sim 90 \%$ prevalence of lactose malabsorption at $20 \mathrm{~g}$ and $40 \mathrm{~g}$ lactose respectively, in both healthy controls and IBS patients ${ }^{5}$, (ii) approximately 50\% prevalence in gastroenterology clinics (data on file, Sir Run Run Shaw Hospital). Based on this data, if lactose intolerance is self-reported by half of IBS patients, then inclusion of 600 hospital patients (after correction for co-morbidity) provides $>80 \%$ power of detecting an increase in IBS symptoms associated with dietary lactose compared to controls at a risk ratio of 2:1 $(\mathrm{p}<0.05)$. For the mechanistic lactose breath tests performed in the hospital population, and assuming $40 \%$ placebo response (typical in functional bowel disease), ${ }^{22}$ this study design has a $80 \%$ power to detect a $20 \%$ difference in prevalence of lactose intolerance following $20 \mathrm{~g}$ lactose challenge between groups.

All statistical analyses were performed using the Statistical Package for Social Sciences for Windows (SPSS16.0 for Windows). Student's t-test was used to compare means and Mann-Whitney U test was used for non-parametric statistical test. Qualitative data comparisons used the chi-square test. Mixed model analysis was used to compare lactose 
consumption from diary and non-dairy foods. All variables were expressed as mean \pm s.d. or median with quartiles as appropriate. Alpha $<0.05$ was considered significant.

\section{RESULTS}

A total of 1022 patients with lower digestive tract symptoms without alarm symptoms attending gastrointestinal clinic of Sir Run Run Shaw hospital were recruited during the study period. 112 patients were excluded due to the presence of organic disease or incomplete questionnaire responses. Thus, results from 910 (89.0\%) outpatient patients were analyzed.

\section{Study participant characteristics and prevalence of SLI}

The prevalence of SLI in clinic patients was $45.2 \%$ (411/910). The prevalence of IBS in SLI subjects was significantly higher than in non-SLI subjects $(61.3 \%(252 / 411)$ vs. $47.3 \%$ (236/499), $\mathrm{p}<0.001)$. The socio-demographic characteristics of subjects with and without SLI are detailed in Table 1. Patients with SLI were younger, had a higher educational level and a higher family income level than those without $\mathrm{SLI}(\mathrm{p}<0.05)$.

\section{Psychiatric state, Life Events Stress and quality of life}

In clinic patients, the level of anxiety and depression was high, with nearly 1 in 5 subjects reporting anxiety or depression. 32.6\% SLI patients and 32.9\% non-SLI subjects had psychological disease (Table 1). There was no difference in the prevalence of psychological disease or life event stress between the two groups (Table 2). Subjects with SLI had a lower 
quality of life than those without SLI as assessed by SF-8 score (including total items and physical but not emotional items). (Table 3)

\section{Objective and self-reported markers of lactose intolerance}

Patients that attended gastroenterology clinic in the morning completed a $20 \mathrm{~g}$ lactose HBT (492/910 (54\%) of total) of whom 232 (47\%) self reported lactose intolerance (SLI). Patient selection was determined by time of clinic attendance only and there was no difference in clinical or other characteristics between patients that completed HBT and those that did not (data on file).

Lactose Malabsorption (definition in methods) was confirmed by an increase in breath hydrogen in $374(76 \%)$ and abdominal symptoms of lactose intolerance were reported by 285/492 (58\%). Patients with irritable bowel syndrome defined by the Rome III criteria had the same rate of lactose malabsorption to other patients but tended to report lactose intolerance during HBT more frequently (61\% (161/264) vs. 54\% (124/228), p=0.14) (Figure 1). With HBT taken as the diagnostic standard, the sensitivity, specificity, positive predictive value (PPV) and negative predictive value (NPV) of SLI was 48.8\%, 55.1\%, 59.9\% and $43.9 \%$, respectively.

A large sample of clinic patients $(n=640)$ and reference data from healthy volunteers that had completed 20g HBT ( $\mathrm{n}=82)$ underwent genetic sequencing of the lactase gene promoter region. The T-13910 allele was not detected in any individual. The genotype in all participants was C/C-13910 (Figure 2). Several novel SNPs were identified in the same region, including C/T-13908 ( $\mathrm{n}=1), \mathrm{A} / \mathrm{C}-13926(\mathrm{n}=2)$ and G/A-14010 (n=2). Only the 
C/T-13908 genotype appeared to confer lactase persistence with a negative 20g lactose HBT (no $\mathrm{H} 2$ production and no symptoms). No significant association was present.

\section{Intake of lactose from dairy and non-dairy source}

In hospital populations subjects that reported SLI ingested less lactose from dairy products than those without SLI ( $\mathrm{p}=0.032)$; however lactose intake from non-dairy sources (i.e. commercially processed foods) was similar (Table 4).

Intake of major food groups and also alcohol was similar in SLI and non-SLI subjects. Further, when foods containing poorly absorbed, fermentable carbohydrates and related substances (i.e. FODMAPs) were considered, patients with and without SLI reported broadly similar food choices on the FFQ (Table 5). In general, where differences existed between groups (e.g. Zucchini), the amounts eaten were small; however, subjects with SLI ate significantly less legumes (e.g. beans, lentils) and dried fruit compared to individuals that did not self-report food intolerance.

\section{DISCUSSION}

The prevalence of SLI was high $(45 \%)$ in a large cohort of patients attending a gastroenterology clinic for investigation and treatment of functional digestive symptoms. Previous surveys documented self-reported lactose intolerance in $10-25 \%$ of the community and in $22-28 \%$ of patients with irritable bowel syndrome. ${ }^{2,5,23}$ Clearly higher rates of food intolerance in this study reflect the population studied, the setting in a gastrointestinal clinic and, very likely, increased intake of dairy products and awareness of lactose intolerance as a 
cause of abdominal symptoms in China. ${ }^{24}$

About half of clinic patients had SLI; however, the prevalence of primary lactase deficiency in the South Chinese population approaches $100 \% .^{5,25}$ The discrepancy between the "genetic biomarker" and "self-reported clinical impact" of this condition can be explained by the interaction between the amount of lactose in the diet and individual sensitivity to the products of lactose fermentation. ${ }^{5,} 10$ In healthy individuals $70-80 \%$ report abdominal symptoms after ingestion of 40-50g lactose (equivalent to 1 liter of milk); however, symptoms are much less common at lower doses (3\% at $10 \mathrm{~g}$ and $22 \%$ at $20 \mathrm{~g}$ lactose). ${ }^{5}$ The average daily intake of lactose by clinic patients in China was $2.0 \mathrm{~g}$ with only $5.8 \%$ taking more than $10.0 \mathrm{~g}$ per day. At these low levels even IBS patients rarely experience symptoms. ${ }^{5}$ Nevertheless, some patients with SLI base their opinion on the occurrence of symptoms after taking a large quantity of milk drinks or ice cream on one particular occasion, and others may wrongly attribute symptoms to unfamiliar or unpalatable dairy products in the past.

Genetic studies of primary lactase deficiency have identified a common single-nucleotide polymorphism (SNP) in the promoter region 13910 base pairs upstream from the structural gene coding for lactase on chromosome $2 \mathrm{q} \cdot 21-22 .^{21}$ This polymorphism consists of the nucleotide switch of $\mathrm{T}$ for C, resulting in variants CC, CT or TT-13910. Enattah and other authors ${ }^{26-30}$ have shown that CC-13910 is a good predictor for loss of intestinal lactase activity and a genetic test of C/T (-13910) polymorphism can be used as a first stage screening test for adult-type hypolactasia. We tested 640 clinic patients and 82 healthy volunteers in the Chinese population. All had the C/C-13910 genotype. A small number of novel SNPs in the same region were identified; however only one individual with 
genotype C/T-13908 (i.e. 2 base pairs from the common SNP) had lactase persistence on lactose HBT. This is a random SNP in a single individual and provides no evidence that the ability to digest lactose confers a selective advantage in the Chinese population. Certainly, consistent with other studies in non-European countries, ${ }^{31}$ gene testing of the C/T (-13910) polymorphism should not be used in screening for lactase persistence in this setting. The lack of genotype-phenotype association also confirms that genetic testing does not predict lactose intolerance at doses found in the normal diet.

Factors associated with self-reported lactose intolerance to dairy products were identified from responses to questionnaires. SLI was not associated with anxiety, depression or life event stress in clinic patients in the present study. This is consistent with results reported by Bohn et al. from a clinical study in Europe, ${ }^{13}$ but contrasts with the results from a large population based study in the Hangzhou population $(n=2000)$ that did show higher rates of SLI in individuals with psychiatric disease and / or high levels of stress (unpublished data). The explanation for this somewhat unexpected finding may be the generally high rates of both physical and psychiatric disease in clinic attendees. ${ }^{32}$ Prospective trials have shown that the presence of psychosocial problems and physical co-morbidity increase the risk of developing post-infectious IBS after an attack of food poisoning. ${ }^{33-35}$ The same factors also increase the risk of food intolerance during a blinded lactose hydrogen breath test (HBT). ${ }^{5,10}$ This study adds "the patient's perspective" to this physiological data. The similarity of food intolerance and IBS symptoms suggest that these conditions may well share a common pathophysiology. ${ }^{36}$ Experimental and clinical evidence have shown that intolerance to a range of poorly absorbed, fermentable carbohydrates (FODMAPs (i.e. Fermentable Oligo-, Di-, and 
Mono-saccharides And Polyols (includes lactose in patients with lactase deficiency)) and indigestible fiber can be the cause of functional abdominal symptoms. ${ }^{37-39}$ Lactose, may be considered a "facultative" FODMAP, as the dose reaching the distal small bowel and colon depends on enzyme activity in the proximal small bowel. Physiological studies have shown that individuals affected by psychosocial problems and those with functional gastrointestinal disease are hypersensitive to visceral events including the gas production caused by lactose malabsorption. ${ }^{5}, 10$ The current study provides evidence that patients can also be "hypervigilant" to dietary factors that may cause digestive discomfort.

The impact of SLI on the diet was evident in clinic patients. Individuals with SLI consumed significantly less lactose in the form of milk, yoghurt, cream and ice cream than other subjects. However, this effect did not extend to a reduction in lactose intake from non-dairy products (e.g. biscuits, sausage and other commercially processed foods) in which lactose content may not be obvious. The impact of SLI on the intake of other foods was less consistent; however, reductions in legumes and dried fruit were noted. This is of interest because these items are well-known to cause bloating and flatulence. Thus, it appears that individuals with SLI restrict intake of food and drink known to cause abdominal symptoms, but not of other substances that also have high levels of fermentable, poorly absorbed carbohydrates but are less well-known to cause problems such as wheat and soft drinks containing fructose. ${ }^{36}$

The inconsistency of food choices provides further evidence that most people find it difficult to identify specific items that cause digestive symptoms. This was evident even for lactose intake in subjects with lactase deficiency (i) by the very weak positive predictive 
value present between SLI and the occurrence of abdominal symptoms during $20 \mathrm{~g}$ lactose HBT and, (ii) by the fact that only SLI and not lactose intolerance on HBT predicted reduced intake of dairy products. This may explain why IBS patients rarely succeed in controlling their condition by self-directed restriction of dairy products. ${ }^{40}$ Rather dietary advice and support provided by a dietician is required to reduce the foods that can trigger functional abdominal symptoms and to ensure that a health, balanced diet is maintained. ${ }^{39,41-43}$

The strengths of this study include the large, representative sample of subjects recruited in gastroenterology clinic with assessment of demographic, clinical, psychosocial and dietary information using validated questionnaires. Further, performance of the study in a Chinese population with $100 \%$ prevalence of lactase deficiency facilitated interpretation of the results since all subjects shared the same "genetic risk" of lactose malabsorption and intolerance. Conversely, the limitation of studying SLI in China is that the average intake of dairy products is lower than in Europe and the USA. This may impact on the generalizability of the findings; although, similar findings for SLI prevalence and its impact on dairy intake have been reported in Western populations. ${ }^{16,23}$

In conclusion, in a population with lactase deficiency, SLI was present almost 1 in 2 patients attending clinic for investigation of functional digestive symptoms. SLI did not predict the occurrence of symptoms by $20 \mathrm{~g}$ Lactose HBT and it was subjective perception of food intolerance and not objective malabsorption that influenced food choices. SLI subjects avoided lactose intake from dairy products and reduced intake also of foods, such as legumes and dried fruit, known to cause bloating and flatulence. Future studies are required to assess whether dietary restriction impacts on the general and nutritional health of these individuals. 


\section{ACKNOWLEDGMENTS}

Guarantor of the article: Ning Dai, Mark Fox. The authors are very grateful for the statistical support provided by Yunxian Yu.

\section{FUNDING}

This work was funded by, but independent of, Nestle Research International (No: 120100047) and the Science and Technology Department of Zhejiang Province, China (No: 2009C14016). This study was also supported by a grant from the National Natural Science Foundation of China (No: 81200274).

\section{COMPETING INTERESTS}

The authors have no competing interests.

\section{AUTHOR CONTRIBUTION}

X.Z. conducted research, analyzed data and drafted the article; H.C., Y.C. and Y.D. conducted research; Y.L. and Y.Z. analyzed data; D.P. designed of the study and performance of study procedures; M.Fr. contributed to the design of the study; N.D. designed the protocol, planned and coordinated the study. M.Fo. wrote the original protocol, provided statistical support and interpretation of the data, and edited the manuscript; M.Fo. and N.D. had primary responsibility for final content. All authors read and approved the final manuscript. 


\section{REFERENCES}

1. Lomer MC, Parkes GC, Sanderson JD. Review article: lactose intolerance in clinical practice--myths and realities. Alimentary pharmacology \& therapeutics 2008;27:93-103.

2. Misselwitz B, Pohl D, Fruhauf H, Fried M, Vavricka SR, Fox M. Lactose malabsorption and intolerance: pathogenesis, diagnosis and treatment. United European gastroenterology journal 2013;1:151-9.

3. Pohl D, Savarino E, Hersberger M, Behlis Z, Stutz B, Goetze O, Eckardstein AV, Fried M, et al. Excellent agreement between genetic and hydrogen breath tests for lactase deficiency and the role of extended symptom assessment. Br J Nutr 2010;104:900-7.

4. Hovde O, Farup PG. A comparison of diagnostic tests for lactose malabsorption--which one is the best? BMC Gastroenterol 2009;9:82.

5. Yang J, Deng Y, Chu H, Cong Y, Zhao J, Pohl D, Misselwitz B, Fried M, et al. Prevalence and presentation of lactose intolerance and effects on dairy product intake in healthy subjects and patients with irritable bowel syndrome. Clinical gastroenterology and hepatology : the official clinical practice journal of the American Gastroenterological Association 2013;11:262-68 e1.

6. Vesa TH, Korpela RA, Sahi T. Tolerance to small amounts of lactose in lactose maldigesters. The American journal of clinical nutrition 1996;64:197-201.

7. Suarez FL, Savaiano DA, Levitt MD. A comparison of symptoms after the consumption of milk or lactose-hydrolyzed milk by people with self-reported severe lactose intolerance. The New England journal of medicine 1995;333:1-4.

8. Casellas F, Aparici A, Casaus M, Rodriguez P, Malagelada JR. Subjective perception of lactose intolerance does not always indicate lactose malabsorption. Clinical gastroenterology and hepatology : the official clinical practice journal of the American Gastroenterological Association 2010;8:581-6.

9. Suchy FJ, Brannon PM, Carpenter TO, Fernandez JR, Gilsanz V, Gould JB, Hall K, Hui SL, et al. National Institutes of Health Consensus Development Conference: lactose intolerance and health. Annals of internal medicine 2010;152:792-6. 
10. Zhu Y, Zheng X, Cong Y, Chu H, Fried M, Dai N, Fox M. Bloating and distention in irritable bowel syndrome: the role of gas production and visceral sensation after lactose ingestion in a population with lactase deficiency. The American journal of gastroenterology 2013;108:1516-25.

11. Zhao J, Fox M, Cong Y, Chu H, Shang Y, Fried M, Dai N. Lactose intolerance in patients with chronic functional diarrhoea: the role of small intestinal bacterial overgrowth. Aliment Pharmacol Ther 2010;31:892-900.

12. Barrett J GP. Clinical ramifications of malabsorption of fructose and other short-chain carbohydrates. Practical Gastroenterology 2007:51-63.

13. Bohn L, Storsrud S, Tornblom H, Bengtsson U, Simren M. Self-reported food-related gastrointestinal symptoms in IBS are common and associated with more severe symptoms and reduced quality of life. Am J Gastroenterol 2013;108:634-41.

14. Kull M, Kallikorm R, Lember M. Impact of molecularly defined hypolactasia, self-perceived milk intolerance and milk consumption on bone mineral density in a population sample in Northern Europe. Scand J Gastroenterol 2009;44:415-21.

15. Obermayer-Pietsch BM, Bonelli CM, Walter DE, Kuhn RJ, Fahrleitner-Pammer A, Berghold A, Goessler W, Stepan V, et al. Genetic predisposition for adult lactose intolerance and relation to diet, bone density, and bone fractures. J Bone Miner Res 2004;19:42-7.

16. Nicklas TA, Qu H, Hughes SO, He M, Wagner SE, Foushee HR, Shewchuk RM. Self-perceived lactose intolerance results in lower intakes of calcium and dairy foods and is associated with hypertension and diabetes in adults. The American journal of clinical nutrition 2011;94:191-8.

17. Holmes TH, Rahe RH. The Social Readjustment Rating Scale. Journal of psychosomatic research 1967;11:213-8.

18. Yuexin Y. Food composition table in China (second volume). Beijing, China: Peking University Medical 2004.

19. Di Stefano M, Miceli E, Mazzocchi S, Tana P, Moroni F, Corazza GR. Visceral hypersensitivity and intolerance symptoms in lactose malabsorption. Neurogastroenterology and motility : the official journal of the European Gastrointestinal Motility Society 2007;19:887-95. 
20. Tishkoff SA, Reed FA, Ranciaro A, Voight BF, Babbitt CC, Silverman JS, Powell K, Mortensen HM, et al. Convergent adaptation of human lactase persistence in Africa and Europe. Nature genetics 2007;39:31-40.

21. Enattah NS, Sahi T, Savilahti E, Terwilliger JD, Peltonen L, Jarvela I. Identification of a variant associated with adult-type hypolactasia. Nature genetics 2002;30:233-7.

22. Spiller RC. Problems and challenges in the design of irritable bowel syndrome clinical trials: experience from published trials. The American journal of medicine 1999;107:91S-97S.

23. Saberi-Firoozi M, Khademolhosseini F, Mehrabani D, Yousefi M, Salehi M, Heidary ST. Subjective lactose intolerance in apparently healthy adults in southern Iran: Is it related to irritable bowel syndrome? Indian journal of medical sciences 2007;61:591-7.

24. Rohrer F. China drinks its milk BBC News Magazine [serial online] 2007. Accessed 7.8.07.

25. Yang Y, He M, Cui H, Bian L, Wang Z. The prevalence of lactase deficiency and lactose intolerance in Chinese children of different ages. Chinese medical journal 2000;113:1129-32. 26. Rasinpera H, Savilahti E, Enattah NS, Kuokkanen M, Totterman N, Lindahl H, Jarvela I, Kolho KL. A genetic test which can be used to diagnose adult-type hypolactasia in children. Gut 2004;53:1571-6.

27. Hogenauer C, Hammer HF, Mellitzer K, Renner W, Krejs GJ, Toplak H. Evaluation of a new DNA test compared with the lactose hydrogen breath test for the diagnosis of lactase non-persistence. European journal of gastroenterology \& hepatology 2005;17:371-6.

28. Ridefelt P, Hakansson LD. Lactose intolerance: lactose tolerance test versus genotyping. Scand J Gastroenterol 2005;40:822-6.

29. Bulhoes AC, Goldani HA, Oliveira FS, Matte US, Mazzuca RB, Silveira TR. Correlation between lactose absorption and the C/T-13910 and G/A-22018 mutations of the lactase-phlorizin hydrolase (LCT) gene in adult-type hypolactasia. Brazilian journal of medical and biological research $=$ Revista brasileira de pesquisas medicas e biologicas $/$ Sociedade Brasileira de Biofisica [et al] 2007;40:1441-6.

30. Kerber M, Oberkanins C, Kriegshauser G, Kollerits B, Dossenbach-Glaninger A, Fuchs D, Ledochowski M. Hydrogen breath testing versus LCT genotyping for the diagnosis of 
lactose intolerance: a matter of age? Clinica chimica acta; international journal of clinical chemistry 2007;383:91-6.

31. Ingram CJ, Elamin MF, Mulcare CA, Weale ME, Tarekegn A, Raga TO, Bekele E, Elamin FM, et al. A novel polymorphism associated with lactose tolerance in Africa: multiple causes for lactase persistence? Human genetics 2007;120:779-88.

32. Drossman DA, Whitehead WE, Toner BB, Diamant N, Hu YJ, Bangdiwala SI, Jia H. What determines severity among patients with painful functional bowel disorders? The American journal of gastroenterology 2000;95:974-80.

33. Mearin F, Rey E, Balboa A. [Functional and motility gastrointestinal disorders]. Gastroenterologia y hepatologia 2012;35 Suppl 1:3-11.

34. Mearin F, Balboa A. [Post-infectious functional gastrointestinal disorders: from the acute episode to chronicity]. Gastroenterologia y hepatologia 2011;34:415-21.

35. Ghoshal UC, Park H, Gwee KA. Bugs and irritable bowel syndrome: The good, the bad and the ugly. Journal of gastroenterology and hepatology 2010;25:244-51.

36. Gibson PR, Shepherd SJ. Evidence-based dietary management of functional gastrointestinal symptoms: The FODMAP approach. Journal of gastroenterology and hepatology 2010;25:252-8.

37. Shepherd SJ, Parker FC, Muir JG, Gibson PR. Dietary triggers of abdominal symptoms in patients with irritable bowel syndrome: randomized placebo-controlled evidence. Clin Gastroenterol Hepatol 2008;6:765-71.

38. Beyer PL, Caviar EM, McCallum RW. Fructose intake at current levels in the United States may cause gastrointestinal distress in normal adults. Journal of the American Dietetic Association 2005;105:1559-66.

39. Ledochowski M, Widner B, Bair H, Probst T, Fuchs D. Fructose- and sorbitol-reduced diet improves mood and gastrointestinal disturbances in fructose malabsorbers. Scand $\mathbf{J}$ Gastroenterol 2000;35:1048-52.

40. Parker TJ, Woolner JT, Prevost AT, Tuffnell Q, Shorthouse M, Hunter JO. Irritable bowel syndrome: is the search for lactose intolerance justified? Eur J Gastroenterol Hepatol 2001;13:219-25. 
41. Shepherd SJ, Gibson PR. Fructose malabsorption and symptoms of irritable bowel syndrome: guidelines for effective dietary management. J Am Diet Assoc 2006;106:1631-9. 42. Johlin FC, Jr., Panther M, Kraft N. Dietary fructose intolerance: diet modification can impact self-rated health and symptom control. Nutrition in clinical care : an official publication of Tufts University 2004;7:92-7.

43. Goldstein R, Braverman D, Stankiewicz H. Carbohydrate malabsorption and the effect of dietary restriction on symptoms of irritable bowel syndrome and functional bowel complaints. The Israel Medical Association journal : IMAJ 2000;2:583-7. 
Table 1 General demographic, social and medical characteristics of SLI and non-SLI subjects from gastroenterology clinic in Sir Run Run Shaw Hospital

\begin{tabular}{|c|c|c|c|c|}
\hline Variables & Levels & $\begin{array}{c}\text { SLI } \\
(n=411)\end{array}$ & $\begin{array}{c}\text { Non-SLI } \\
(n=499)\end{array}$ & $p$-value \\
\hline Age, yr & Mean \pm SD & $43.0 \pm 12.0$ & $45.1 \pm 11.9$ & 0.010 \\
\hline \multirow[t]{2}{*}{ Sex, $\mathrm{n}(\%)$} & Male & $219(53.3 \%)$ & $284(56.9 \%)$ & \\
\hline & Female & $192(46.7 \%)$ & $215(43.1 \%)$ & 0.273 \\
\hline \multirow[t]{3}{*}{ Marital status, $\mathrm{n}(\%)$} & Married & $361(87.8 \%)$ & $439(88.0 \%)$ & \\
\hline & Single & $37(9.0 \%)$ & $35(7.0 \%)$ & 0.227 \\
\hline & Unmarried & $13(3.2 \%)$ & $25(5.0 \%)$ & \\
\hline \multirow[t]{4}{*}{ Education, $\mathrm{n}(\%)$} & $<=$ primary & $81(19.7 \%)$ & $153(30.7 \%)$ & \\
\hline & Middle school & $100(24.3 \%)$ & $158(31.7 \%)$ & $<0.001$ \\
\hline & High School & $120(29.2 \%)$ & $105(21.0 \%)$ & \\
\hline & $>=$ college & $110(26.8 \%)$ & $83(16.6 \%)$ & \\
\hline \multirow{4}{*}{$\begin{array}{c}\text { Average Family } \\
\text { income } \mathrm{RMB} / \text { month } \\
\mathrm{n}(\%)\end{array}$} & $<1000$ & $34(8.3 \%)$ & $59(11.8 \%)$ & \\
\hline & $1000 \sim$ & $228(55.5 \%)$ & $317(63.5 \%)$ & 0.001 \\
\hline & $5000 \sim$ & $57(13.9 \%)$ & $50(10.0 \%)$ & \\
\hline & $>=10000$ & $92(22.4 \%)$ & $73(14.6 \%)$ & \\
\hline \multirow[t]{4}{*}{ Job, $\mathrm{n}(\%)$} & Office work & $53(12.9 \%)$ & $45(9.0 \%)$ & \\
\hline & Physical work & $235(57.2 \%)$ & $315(63.1 \%)$ & 0.009 \\
\hline & Housework & $104(25.3 \%)$ & $131(26.3 \%)$ & \\
\hline & Student & $19(4.6 \%)$ & $8(1.6 \%)$ & \\
\hline \multirow{4}{*}{$\begin{array}{c}\text { Cigarette Smoking, } \\
\mathrm{n}(\%)\end{array}$} & Never & $306(74.5 \%)$ & $346(69.3 \%)$ & \\
\hline & $1 \sim$ & $50(12.2 \%)$ & $75(15.0 \%)$ & 0.167 \\
\hline & $10 \sim$ & $23(5.6 \%)$ & $23(4.6 \%)$ & \\
\hline & $20 \sim$ & $32(7.8 \%)$ & $55(11.0 \%)$ & \\
\hline \multirow{4}{*}{$\begin{array}{c}\text { Alcohol Drinking, } \\
\mathrm{n}(\%)\end{array}$} & Never & $183(44.5 \%)$ & $222(44.5 \%)$ & \\
\hline & Some time & $173(42.1 \%)$ & $170(34.1 \%)$ & 0.005 \\
\hline & Often & $43(10.5 \%)$ & $90(18.0 \%)$ & \\
\hline & Always & $12(2.9 \%)$ & $17(3.4 \%)$ & \\
\hline \multirow{2}{*}{$\begin{array}{c}\text { Medical History, } \\
\mathrm{n}(\%)\end{array}$} & No & $218(53.0 \%)$ & $261(52.3 \%)$ & 0.825 \\
\hline & Yes & $193(47.0 \%)$ & $238(47.7 \%)$ & \\
\hline Psychological & No & $277(67.4 \%)$ & $335(67.1 \%)$ & 0.933 \\
\hline $\begin{array}{c}\text { Disease (HADS >8) } \\
\mathrm{n}(\%)\end{array}$ & Yes & $134(32.6 \%)$ & $164(32.9 \%)$ & \\
\hline
\end{tabular}

HADS, hospital anxiety and depression scale; SLI, self-reported lactose intolerance. 
Table 2 Anxiety, Depression and Life Event Stress reported by SLI and non-SLI subjects from hospital groups

\begin{tabular}{cccc}
\hline & SLI $(\mathrm{n}=411)$ & Non-SLI $(\mathrm{n}=499)$ & $p$-value \\
\hline Anxiety & & & \\
Yes & $98(23.8 \%)$ & $98(19.6 \%)$ & 0.125 \\
No & $313(76.2 \%)$ & $401(80.4 \%)$ & \\
Depression & & & \\
Yes & $105(25.5 \%)$ & $121(24.2 \%)$ & 0.652 \\
No & $306(74.5 \%)$ & $378(75.8 \%)$ & \\
LEST & $51.0(16.0 \sim 105.0)$ & $54.0(16.0 \sim 104.0)$ & 0.762 \\
\hline
\end{tabular}

SLI, self-reported lactose intolerance; LEST, life event stress test.

Table 3 SF-8 scale score in SLI and non-SLI subjects from hospital

\begin{tabular}{cccc}
\hline & SLI $(\mathrm{n}=411)$ & Non-SLI $(\mathrm{n}=499)$ & $p$-value \\
\hline PCS & $45.10 \pm 7.82$ & $46.40 \pm 7.81$ & 0.0035 \\
MCS & $48.11 \pm 7.83$ & $48.44 \pm 8.06$ & 0.6499 \\
TSF8 & $46.50 \pm 5.08$ & $47.25 \pm 4.97$ & 0.0144 \\
\hline
\end{tabular}

SLI, self-reported lactose intolerance; PCS, average score from four physical items; MCS, average score from four mental items; TSF8, average score from eight items.

Table 4 Lactose intake from dairy and non-dairy sources in SLI and non-SLI subjects. $\left(\mathrm{g} \mathrm{month}^{-1}\right)$

\begin{tabular}{cccc}
\hline & SLI (n=411) & Non-SLI (n=499) & $p$-value \\
\hline Dairy & $13(0-59)$ & $14(0-62)$ & 0.0318 \\
Non-dairy & $24(8-59)$ & $18(5-46)$ & 0.1881 \\
\hline
\end{tabular}

SLI, self-reported lactose intolerance. 
Table 5 Food intake of selected foods and beverages from FFQ in SLI and non-SLI subjects from hospital groups (kg month ${ }^{-1}$ )

\begin{tabular}{|c|c|c|c|}
\hline & $\operatorname{SLI}(n=411)$ & Non-SLI $(\mathrm{n}=499)$ & $p$-value \\
\hline Apple & $1.2(0.4-1.6)$ & $1.2(0-1.2)$ & 0.125 \\
\hline Pear & $0(0-0.8)$ & $0(0-0.8)$ & 0.247 \\
\hline Mandarin & $0(0-1.2)$ & $0.4(0-1.2)$ & 0.316 \\
\hline Apricot & $0(0-0)$ & $0(0-0)$ & 0.369 \\
\hline Peaches & $0(0-0)$ & $0(0-0)$ & 0.676 \\
\hline Grapes & $0(0-0.8)$ & $0(0-0.4)$ & 0.075 \\
\hline Watermelon & $0.8(0-1.2)$ & $0.4(0-1.2)$ & 0.658 \\
\hline Dried fruit & $0(0-0.4)$ & $0(0-0.4)$ & 0.052 \\
\hline Carbonated & $0(0-1.2)$ & $0(0-1.6)$ & 0.429 \\
\hline Honey & $0(0-0)$ & $0(0-0)$ & 0.527 \\
\hline Wine & $0(0-0)$ & $0(0-0)$ & 0.722 \\
\hline Onion & $0(0-0.4)$ & $0(0-0.4)$ & 0.117 \\
\hline Cabbage & $0.8(0.4-1.2)$ & $0.8(0-1.2)$ & 0.038 \\
\hline Artichoke & $0(0-0)$ & $0(0-0)$ & 0.505 \\
\hline Zucchini & $0(0-0.8)$ & $0(0-0.4)$ & 0.051 \\
\hline Legumes & 1.0(0.8-3.0) & $1.2(0.8-3.0)$ & 0.037 \\
\hline Noodles & $1.2(0.8-2.0)$ & $1.2(0.8-2.4)$ & 0.518 \\
\hline Gum & $0(0-0)$ & $0(0-0)$ & 0.464 \\
\hline
\end{tabular}

SLI, self-reported lactose intolerance. Values were expressed as median with 25th and 75 th quartiles. 


\section{Figure 1}

Relationship between subjective and objective lactose tolerance in study population

Clinic population with lactose deficiency and functional GI symptoms and completed breath test ( $n=492$ of total 910 attendees)

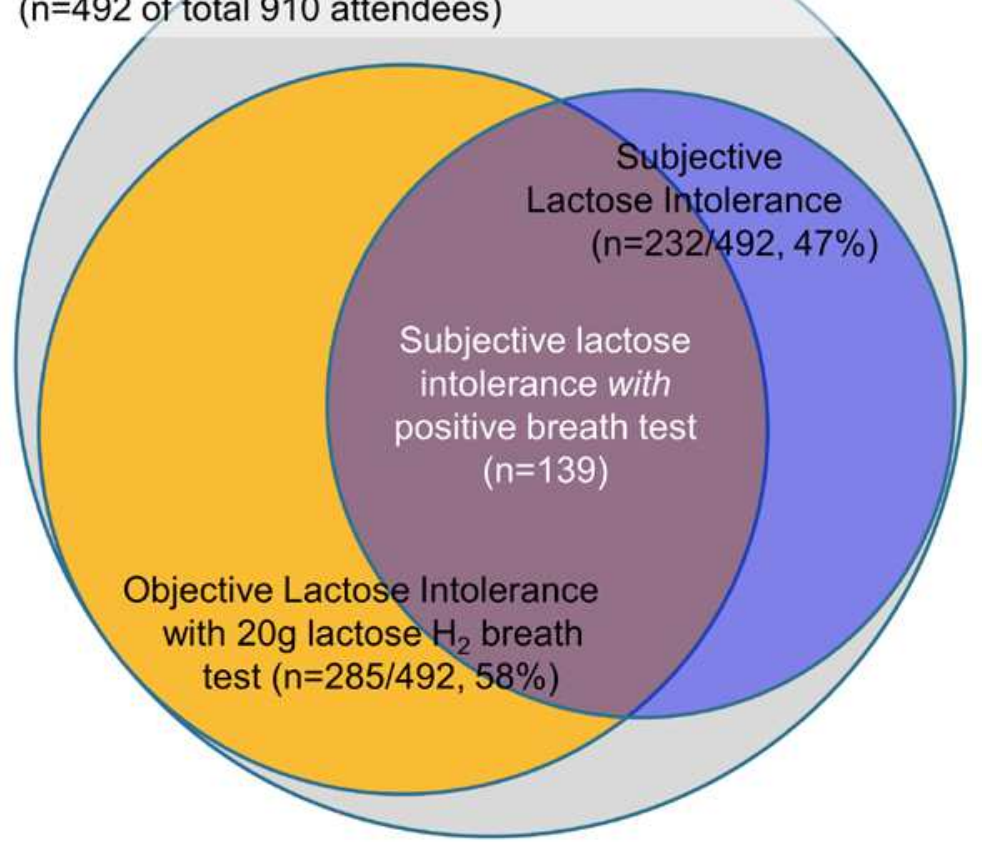

